\title{
A photon pressure calibrator for the GEO 600 gravitational wave detector
}

\author{
K. Mossavi*, M. Hewitson, S. Hild, F. Seifert, U. Weiland, J.R. Smith, H. Lück, H. Grote, \\ B. Willke, K. Danzmann
}

Max-Planck-Institut für Gravitationsphysik (Albert-Einstein-Institut) und Universität Hannover, Callinstr. 38, 30167 Hannover, Germany

Received 7 November 2005; received in revised form 20 December 2005; accepted 21 December 2005

Available online 28 December 2005

Communicated by P.R. Holland

\begin{abstract}
Interferometer mirror displacement induced by radiation pressure is used to demonstrate an alternative calibration method for the GEO 600 detector. The photon calibrator utilizes an amplitude modulated laser diode with up to $1.4 \mathrm{~W}$ output power at a wavelength of $1035 \mathrm{~nm}$. The achieved accuracy of the strain amplitude calibration is dominated by the laser power calibration error, which is in the range of $\pm 4 \%$ for the measurements presented in this Letter.
\end{abstract}

(C) 2005 Elsevier B.V. All rights reserved.

PACS: 04.80.Nn; 42.55.Px; 42.88.+h

Keywords: Gravitational wave detector; Calibration; Radiation pressure; Laser diode

\section{Introduction}

In recent years we have witnessed extensive progress in the development of long base-line interferometric gravitational wave detectors [1-4]. Accurate and reliable calibration of these detectors is of great importance for detector characterization and the extraction of (astro-)physical results.

The current method used to calibrate the output signal of the dual-recycled German-British detector GEO 600 to strain is based on the injection of calibration signals into the electrostatic length-control actuator. The details, development and application of this calibration scheme are given in $[5,6]$. At present, no device is available to evaluate the performance and long-term stability of the current calibration method. In Ref. [7] it was shown that radiation pressure supplied by an amplitude modulated Nd:YAG nonplanar ring oscillator provides a simple and physically independent method for calibrating interferometric gravitational wave detectors.

In this work we describe the implementation of a photon pressure calibrator into the GEO 600 detector by means of a

\footnotetext{
* Corresponding author. Tel.: +49 511762 4780; fax: +49 5117622784 .

E-mail address: mossavi@aei.mpg.de (K. Mossavi).
}

current modulated laser diode. The photon pressure calibrator is currently the only available instrument to validate the absolute calibration of GEO 600.

\section{Experimental arrangement}

The experimental setup for measurements with the photon calibrator together with a section of the GEO 600 detector is shown in Fig. 1. The calibrator consists of a fiber-coupled laser diode (Frankfurt Laser Company) with integrated thermoelectric cooler and power monitor photodiode. The laser diode can generate up to $1.4 \mathrm{~W}$ output power at a wavelength of $1035 \mathrm{~nm}$. This wavelength lies within the maximum reflectivity range of interferometer mirror $\mathrm{M}$. The transmission curve supplied with the mirror allows to estimate a reflectivity of $>99.99 \%$ at $1035 \mathrm{~nm}$. All optical components in the north building of GEO 600 that form part of the interferometer control system, and that could be exposed to light from the laser diode, were isolated using $1064 \mathrm{~nm}$ narrow bandpass interference filters. An $f=60 \mathrm{~mm}$ lens (L) was used to collimate the diverging output of the optical fiber to a Gaussian-like beam with a diameter of $6 \mathrm{~mm}$ at mirror $\mathrm{M}$. To avoid back reflection of light into the laser diode, the collimated beam hits $\mathrm{M}$ under an an- 
gle of $\alpha=2.2^{\circ}$ in a plane parallel to ground. Antireflective coatings were used on all optics in the path of the calibrator beam to minimize power losses. The residual reflectivities for the viewport and the collimator lens at $1035 \mathrm{~nm}$ are $0.14 \%$ and $0.12 \%$ according to the reflectivity curves provided by the coating vendors. A signal generator connected to the laser diode driver (ILX Lightwave, model LDC-3744B) modulates the laser diode current and thus the laser power at an angular frequency $\omega_{m}$.

Treating mirror $\mathrm{M}$ as a single suspended pendulum with an angular resonance frequency much lower than $\omega_{m}$, the magnitude of the longitudinal mirror displacement $x\left(\omega_{m}\right)$ due to

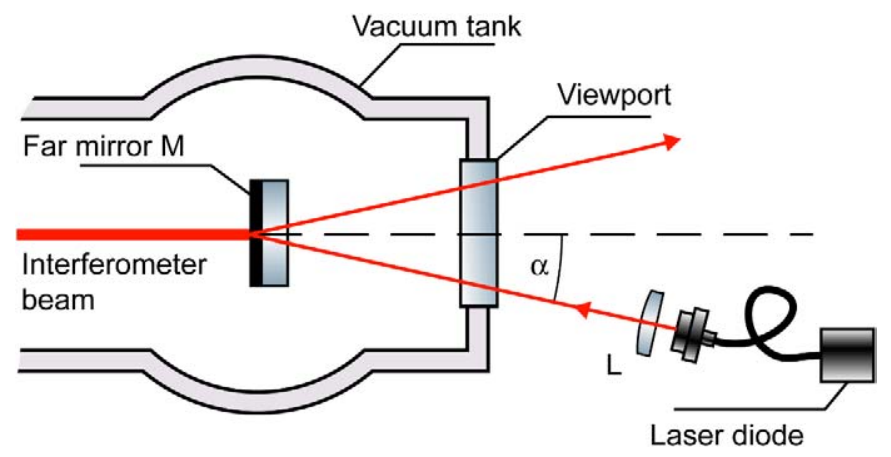

Fig. 1. Experimental arrangement for measurements with the photon calibrator. M: Far mirror in the north building of the GEO 600 detector. Illumination of this mirror with light from the laser diode produces a differential arm-length change that is measured at the main output of the interferometer. radiation pressure is given by [7]

$x\left(\omega_{m}\right)=\frac{2 P_{m} \cos (\alpha)}{M c \omega_{m}^{2}}$,

where $P_{m}$ is the laser power at $\omega_{m}$ and $M=5.32 \pm 0.02 \mathrm{~kg}$ is the mass of the mirror.

The internal photodiode of the laser diode was calibrated by a laser power meter (Coherent LM-3) with an accuracy of $\pm 4 \%$. The power meter was placed behind the fiber output during the calibration process. Due to nonlinearities in the output power versus current characteristics of the laser diode, the modulated laser light contains a certain amount of power at harmonics of $\omega_{m}$ depending on the settings for the laser diode bias current and modulation depth. To account for the harmonic content and thereby improve the calibration accuracy, the laser power at $\omega_{m}$ was derived from the spectrum of the photodiode signal. In addition, the power value was corrected for the losses introduced by the residual reflectivities of the collimator lens and the viewport.

\section{Results}

Fig. 2 shows two consecutive spectra of the detectors strain sensitivity, calibrated with the electrostatic actuator method [6]. The second spectrum was recorded with the laser diode turned on and modulated at $986 \mathrm{~Hz}$ with $P_{m}=284 \mathrm{~mW}(\mathrm{rms})$. The rms mirror displacement due to the radiation pressure corresponds to a value of $x\left(\omega_{m}\right)=9.3 \times 10^{-18} \mathrm{~m}$. In agreement with (1), the photon calibrator signal follows a linear dependence on the laser power (Fig. 3) and a $1 / f^{2}$ dependence on the modulation frequency (Fig. 4).
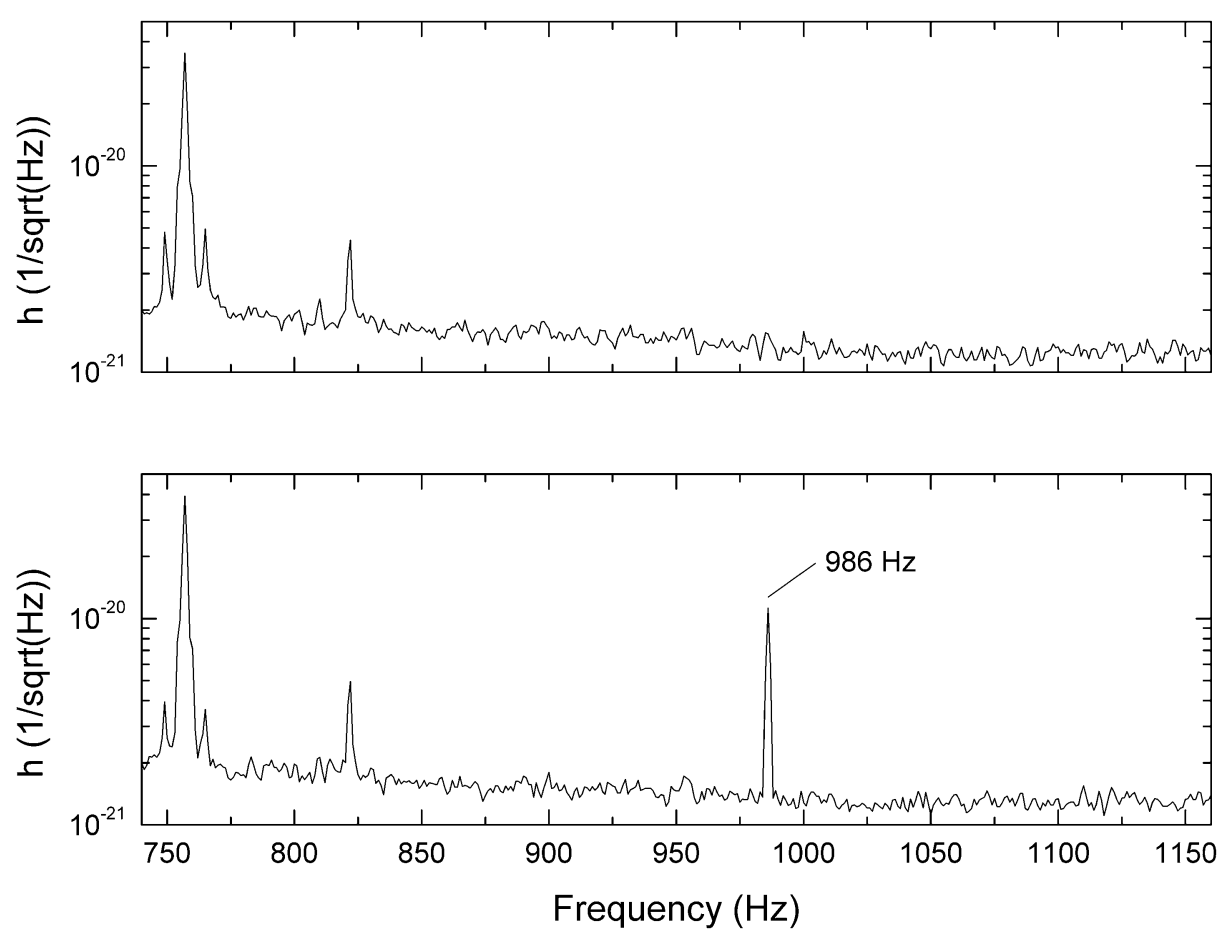

Fig. 2. Section of the GEO strain sensitivity spectrum shortly before and during activation of the photon calibrator with a modulation frequency of $986 \mathrm{~Hz}$. Spectra were created by Fourier transformation of 1-s data segments and 60 averages. Laser diode power: $284 \mathrm{~mW}$ (rms). 


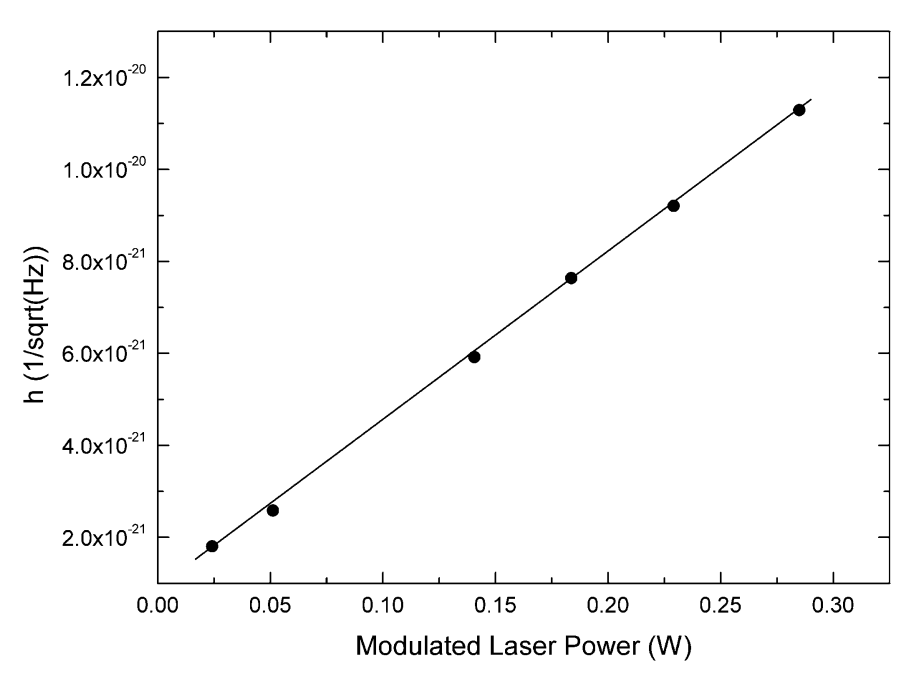

Fig. 3. Strain amplitude versus laser diode power (rms). The solid line indicates a linear dependence. Modulation frequency: $986 \mathrm{~Hz}$.

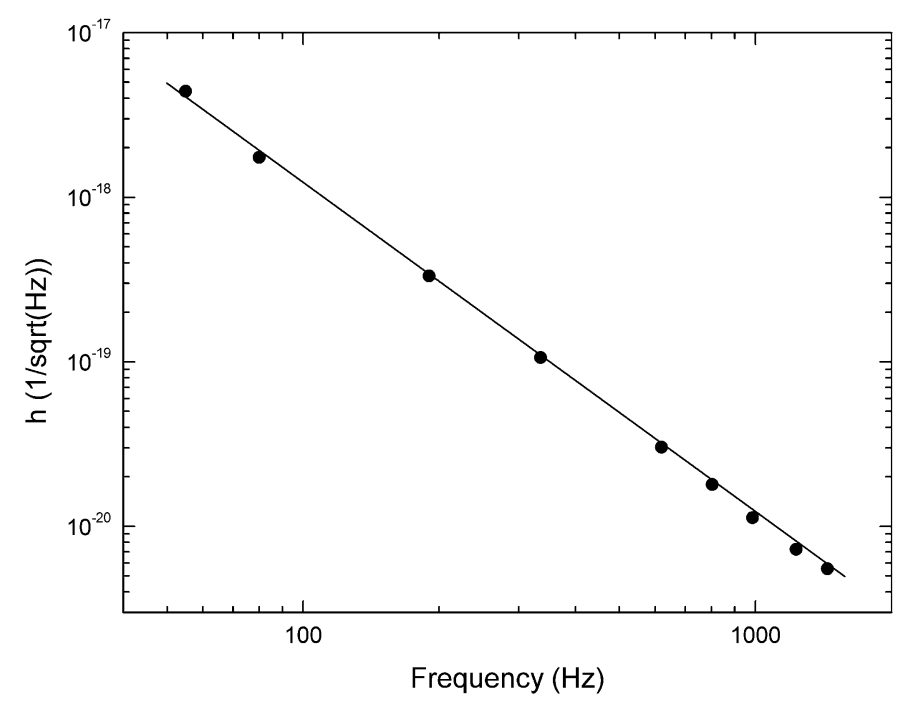

Fig. 4. Frequency dependence of the strain amplitude produced by the photon calibrator. A $1 / f^{2}$ line is shown for reference. Laser diode power: $284 \mathrm{~mW}$ (rms).

A first comparison between the electrostatic actuator and radiation pressure calibration method for the current detector configuration is depicted in Fig. 5. Photon pressure strain values $h_{\text {photon were obtained from (1) and [8] }}$

$h_{\text {photon }}=\frac{2 x\left(\omega_{m}\right)}{L}$,

with the interferometer arm length $L=1200 \mathrm{~m}$. It should be noted that in the case of GEO 600 the factor of 2 in Eq. (2) is preserved, despite the fact that the photon calibrator interacts only with one detector arm, because the far mirror is the folding mirror and is hit twice by the interferome-

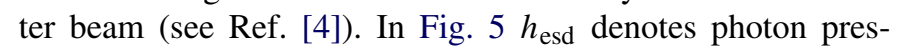
sure induced strain values as measured at the detector output and calibrated by the electrostatic actuator method with a frequency dependent calibration accuracy of a few percent $[9,10]$.

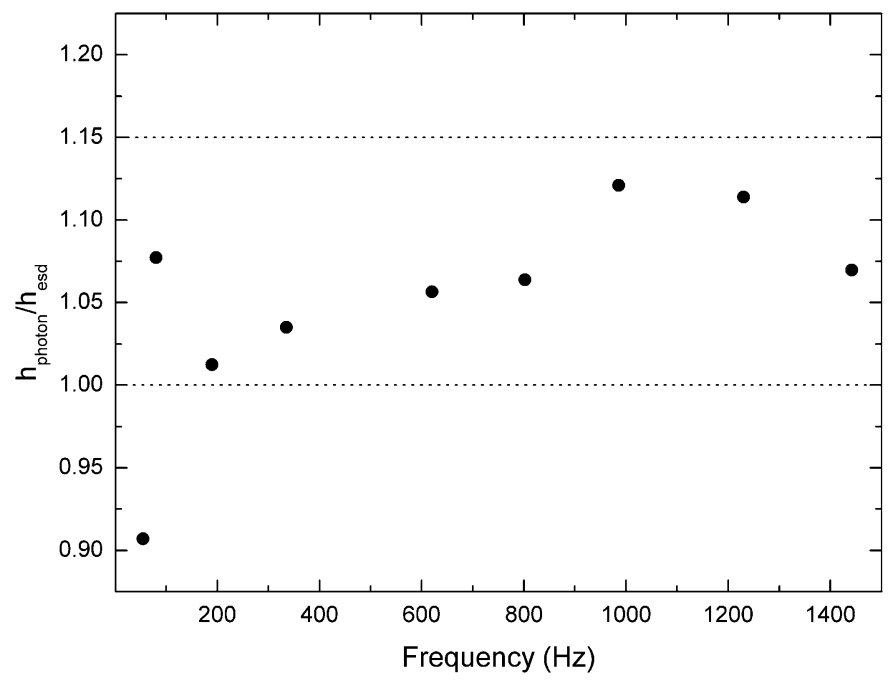

Fig. 5. Comparison between photon pressure $\left(h_{\text {photon }}\right)$ and electrostatic actuator $\left(h_{\text {esd }}\right)$ calibration method.

Fig. 5 indicates a reasonably good agreement between the two calibration methods with a deviation of less than $15 \%$ across the detection band of GEO $600(\sim 100-2000 \mathrm{~Hz})$. It is also obvious from Fig. 5 that strain values produced by the electrostatic actuator calibration method tend to be lower than $h_{\text {photon values. A reproducible opposite tendency was observed }}$ at $55 \mathrm{~Hz}$. Additional measurements are in preparation to investigate the origin of these features.

\section{Conclusions}

We have shown that a current modulated laser diode at $1035 \mathrm{~nm}$ can be used as a compact photon calibrator for interferometric gravitational wave detectors. The measured dependences on the laser diode power and modulation frequency are in good agreement with the theoretical predictions. The accuracy of the photon calibrator is mainly determined by the laser power meter accuracy. A strain calibration accuracy of about $\pm 1 \%$ should be feasible by further reduction of the laser power uncertainty. Further investigations of the discussed calibration methods will be performed to characterize the long term performance and accuracy for different detector operating conditions.

\section{References}

[1] D. Sigg, et al., Class. Quantum Grav. 21 (2004) S409.

[2] F. Acernese, et al., Class. Quantum Grav. 21 (2004) S385.

[3] R. Takahashi, et al., Class. Quantum Grav. 21 (2004) S403.

[4] B. Willke, et al., Class. Quantum Grav. 21 (2004) S417.

[5] M. Hewitson, H. Grote, G. Heinzel, K.A. Strain, H. Ward, U. Weiland, Rev. Sci. Instrum. 74 (2003) 4148.

[6] M. Hewitson, G. Heinzel, J.R. Smith, K.A. Strain, H. Ward, Rev. Sci. Instrum. 75 (2004) 4702.

[7] D.A. Clubley, G.P. Newton, K.D. Skeldon, J. Hough, Phys. Lett. A 283 (2001) 85

[8] É.É. Flanagan, S.A. Hughes, New J. Phys. 7 (2005) 204.

[9] M. Hewitson, S. Babak, R. Balasubramanian, K. Danzmann, H. Grote, G. Heinzel, J. Hough, H. Lück, M.A. Papa, J.R. Smith, K.A. Strain, H. Ward, B. Willke, G. Woan, Class. Quantum Grav. 21 (2004) S1711.

[10] M. Hewitson, H. Grote, S. Hild, H. Lück, P. Ajith, J.R. Smith, K.A. Strain, B. Willke, G. Woan, Class. Quantum Grav. 22 (2005) 4253. 